\title{
NEW RESULTS ABOUT IMPARTIAL SOLITAIRE CLOBBER *
}

\author{
ERic Duchêne ${ }^{1}$, Sylvain Gravier $^{2}$ And Julien Moncel $^{3}$
}

\begin{abstract}
Impartial Solitaire Clobber is a one-player version of the combinatorial game Clobber, introduced by Albert et al. in 2002. The initial configuration of Impartial Solitaire Clobber is a graph, such that there is a stone placed on each of its vertex, each stone being black or white. A move of the game consists in picking a stone, and clobbering an adjacent stone of the opposite color. By clobbering we mean that the clobbered stone is removed from the graph, and replaced by the clobbering one. The aim is to make a sequence of moves leaving the minimum number of stones on the graph; this number is called the reducibility value of the configuration. As any one-player game, Solitaire Clobber is essentially an optimization problem, whose resolution may give bounds on the two-player version of the game. As an optimization problem, Solitaire Clobber can be considered as a constrained version of the underlying optimization problem related to Hamiltonian path. This enables to show that Solitaire Clobber is NP-hard. Solitaire Clobber was already studied in various graph structures, including paths, cycles, trees, and Hamming graphs. In this paper we investigate the problem in complete multipartite graphs. In particular, we give a linear-time algorithm computing the reducibility value of any configuration in complete multipartite graphs. We also address some extremal questions related to Solitaire Clobber in general graphs.
\end{abstract}

Keywords. Combinatorial games, games invoking graphs, extremal problem, solitaire clobber.

Mathematics Subject Classification. 91A46, 91A46, 0SC35.

Received January 25, 2008. Accepted July 8, 2009.

* ERTé "Maths à modeler"

1 ATER, IUFM de Grenoble, France; educhene1@yahoo.fr, educhene@bat710.univ-lyon1.fr

2 CNRS, France; sylvain.gravier@ujf-grenoble.fr

${ }^{3}$ Laboratoire G-SCOP, Grenoble, France; julien.moncel@g-scop.inpg.fr 


\section{Introduction AND DEFINITIONS}

We consider the game Solitaire Clobber defined by Demaine et al. in [5]. This game is actually a variant of the two-player Clobber introduced by Albert et al. in $[1]$.

Solitaire Clobber is a one-player game with the following rules: black and white stones are placed on the vertices of a given graph $G$ (one per vertex). A move consists in picking a stone and clobbering another one of the opposite color located on an adjacent vertex. The clobbered stone is removed from the graph and is replaced by the picked one. The goal is to find a succession of moves that minimizes the number of remaining stones.

In the literature, two versions of Solitaire Clobber coexist: the Partisan and the Impartial ones. In Partisan Solitaire Clobber defined by Demaine et al. [5], the player is forced to alternate black and white captures. In Impartial Solitaire Clobber [6], this constraint does not exist. The present paper deals with the impartial version that we call $I S C$ in its abbreviated form.

A game configuration of $I S C$ is said to be $k$-reducible if there exists a succession of moves that leaves at most $k$ stones on the graph. The reducibility value of a game configuration $C$ is the smallest integer $k$ for which $C$ is $k$-reducible.

As announced in the abstract, this game appears to be an optimization problem on graphs:

\section{Impartial Solitaire Clobber - ISC}

Instance. A configuration $C=(G, \ell)$, where $G$ is a graph, and $\ell: V \longrightarrow\{b, w\}$ is a labelling of the vertices of $G$.

Objective. Minimizing the number of labeled vertices on $G$ by playing allowed moves.

It turns out that Impartial Solitaire ClobBer is a NP-hard problem since the simple question of 1-reducibility of a given configuration can be reduced to the HAMILTONIAN PATH problem. Indeed, if you consider a graph with a black stone on some vertex $v$ and where all the other stones are white, then this configuration is clearly 1-reducible if and only if there exists an Hamiltonian path between $v$ and some other vertex. Since the problem Hamiltonian PATH was proved to be NP-complete on graphs in general, and on grid graphs (i.e., induced subgraphs of the grid) in particular (see [7]), Impartial Solitaire Clobber is a NP-hard problem on these kinds of graphs.

Nevertheless Impartial Solitaire ClobBer was investigated on paths, cycles, and trees (see $[2,3]$ ). The reducibility value of any configuration on a path or a cycle of size $n$ can be computed in linear time $O(n)$. On a tree, there exists an algorithm working in $O\left(n^{3}\right)$ operations. In [6], it was proved that any game configuration on a Hamming graph is 1-reducible, except for hypercubes which are 2-reducible.

For convenience for the reader, we may often mix up a vertex with the stone that it supports. The label or color of a vertex will define the color of the stone located on it. We may also say that "a vertex clobbers another one", instead of talking about the corresponding stones. 


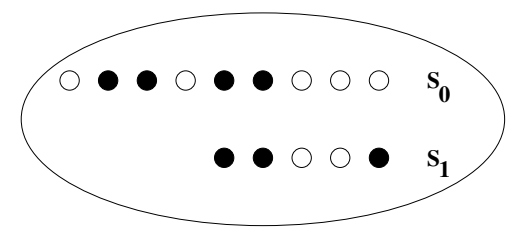

Figure 1. A game configuration on a complete bipartite graph.

Given a game configuration $C$ on a graph $G$, we say that a label/color $c$ is rare on a subgraph $S$ of $G$ if there exists a unique vertex $v \in S$ such that $v$ is labeled $c$. On the contrary, $c$ is said to be common if there exist at least two vertices of this color in $S$. A configuration is said to be monochromatic if all the vertices have the same color. It is said proper otherwise. In all this paper, only proper configurations will be considered.

If $c$ is a color, denote by $\bar{c}$ the opposite color. If $v$ is a vertex of $G$, the color of $v$ will be denoted by $c(v)$.

According to a probabilistic result of Ruszinkó [9], almost all configurations are 1-reducible (in the sense that, for any fixed $p \in] 0,1[$, a configuration on the random graph $G_{n, p}$ is 1-reducible with probability tending to 1 as $n$ tends to infinity - for details about Erdös-Rényi's random graph model $G_{n, p}$, see [4]). This provides us a motivation for the study of configurations which are not 1-reducible. In the next section of this paper, we study the case of complete multipartite graphs and decide when a configuration cannot be 1-reducible. For these graphs, we give a general formula which computes the reducibility value of any game configuration in linear time. In Section 3, we consider extremal questions about $I S C$ : given a graph $G$, we investigate the proper configurations that minimize and maximize the reducibility value on $G$. In [3], it was showed that the reducibility value of a configuration on a cycle $C_{n}$ is at most $\lfloor n / 3\rfloor$. Moreover, for all $n$ there exists a configuration for which this bound is tight. In the present paper, we claim that on any graph with $n$ vertices the reducibility value of a proper configuration is at most equal to $n-\delta$, where $\delta$ is the minimum degree of the graph. Therefore, for any fixed value of $\delta$, we find out the only graphs for which the reducibility value is equal to $n-\delta$.

\section{ISC PLAYED ON COMPLETE MULTIPARTITE GRAPHS}

\subsection{ISC ON COMPLETE BIPARTITE GRAPHS}

Let $G=(V, E)$ be a complete bipartite graph. Therefore, $G$ can be splitted into two stable sets, say $S_{0}$ and $S_{1}$. A game configuration on a complete bipartite graph will be represented by a "two rows" layout, each row refering to a stable set $S_{i}$ with $i \in\{0,1\}$. Figure 1 shows an example of a configuration on a complete bipartite graph. One can move any stone of a row to any stone of the other row having the opposite color. Moves inside a row are not allowed. 
In [5], Demaine et al. define an invariant $\delta$ available for $I S C$ on bipartite graphs. The description of $\delta$ follows.

Let $C$ be a game configuration on a bipartite graph $G$. We allocate the color white the to set $S_{0}$, and the color black to $S_{1}$. A stone of $C$ is said to be clashing if its color differs from the color of the stable set to which it belongs. Denote by $\delta(C)$ the following quantity:

$$
\delta(C)=\text { number of stones }+ \text { number of clashing stones. }
$$

In their paper, Demaine et al. proved that $(\delta(C) \bmod [3])$ never changes during the game, where $a$ mod $[b]$ is the remainder of the division of $a$ by $b$. This implies that any 1-reducible configuration on a bipartite graph satisfies $\delta(C) \not \equiv 0 \bmod [3]$.

A complete bipartite graph is said to be balanced if $S_{0}$ and $S_{1}$ have the same size. It is said well-colored if $S_{0}$ and $S_{1}$ are both monochromatic. In a well-colored configuration, one does not lose generality by assuming that the stones of $S_{0}$ (resp. $S_{1}$ ) are white (resp. black). Under this assumption, there is no clashing stone in a well-colored configuration.

\subsubsection{The reducibility value of balanced complete bipartite graphs}

We now give a first result about the reduciblity value of a well-colored balanced complete bipartite graph.

Lemma 2.1. A well-colored balanced complete bipartite graph is 1-reducible if $\delta \not \equiv 0 \bmod [3]$. Otherwise, the reducibility value is equal to 2.

Proof. Let $C$ be a game configuration on a well-colored balanced complete bipartite graph. Denote by $n$ the size of each stable set. Then $\delta(C)=2 n$. According to the result of Demaine et al., a necessary condition for $C$ to be 1-reducible is that $\delta(C) \not \equiv 0 \bmod [3]$, which means $n \not \equiv 0 \bmod [3]$.

Let $n \geq 4$. Then $C$ can be reduced to a well-colored balanced complete bipartite graph where each stable set has a size equal to $n-3$. The reduction is the following:

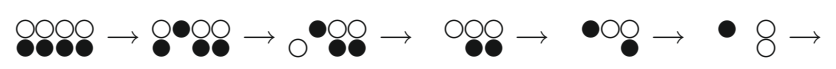

By applying recursively this operation, and according to the value $(n \bmod [3]), C$ can be reduced to one of these three configurations:

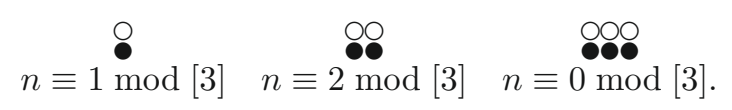

One clearly see that when $n \not \equiv 0$ mod [3], the above configurations are 1-reducible. When $n \equiv 0$ mod [3], the above configuration is 2-reducible and we can not improve this reduction because of the invariant.

Remark 2.1. When $n \not \equiv 0 \bmod [3]$, the value $\delta(C)$ sets a correlation between the color and the location of the remaining stone. When $n \equiv 1 \bmod [3]$, the last stone will be clashing. It will not be the case when $n \equiv 2 \bmod [3]$. 


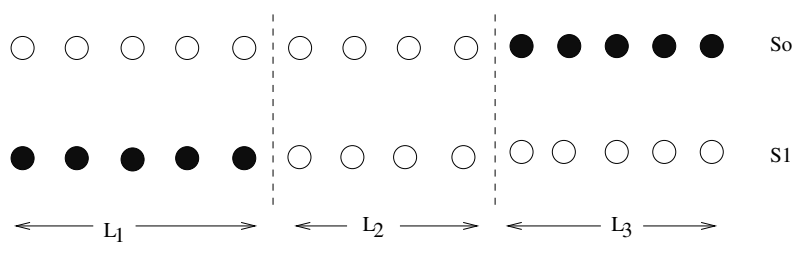

Figure 2. Splitting a balanced complete bipartite graph.

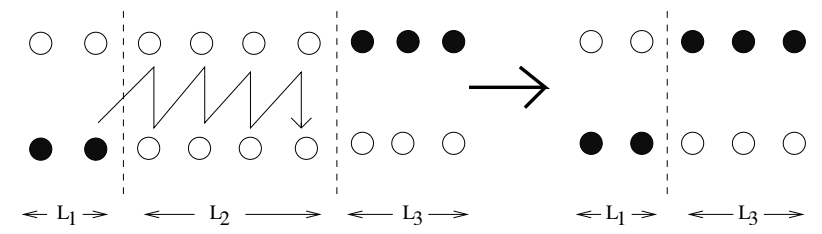

Figure 3. Clobbering the monochromatic part of length $L_{2}$.

Lemma 2.2. A balanced complete bipartite graph is 1-reducible if $\delta \not \equiv 0 \bmod [3]$. Otherwise, the reducibility value is equal to 2.

Proof. For more convenience, we arrange "graphically" $S_{0}$ and $S_{1}$ as follows: on $S_{0}$ the white stones are placed on the left, and the black ones on the right. We proceed conversely on $S_{1}$. This operation splits $G$ into three complete bipartite subgraphs, of respective lengths $L_{1}, L_{2}$ and $L_{3}$, as shown in Figure 2.

The values $L_{1}$ and $L_{3}$ are the lengths of both well-colored balanced parts, whereas $L_{2}$ is dedicated to the monochromatic part.

According to this notation, $\delta(C)=2 L_{1}+3 L_{2}+4 L_{3}$. Hence $\delta(C) \equiv 0 \bmod [3]$ if and only if $L_{1} \equiv L_{3} \bmod [3]$. Without loss of generality, assume that the monochromatic part $L_{2}$ is white. Since there exists a black stone somewhere, we have it clobber the whole monochromatic part of size $L_{2}$, as shown in Figure 3:

We now consider the resulting graph as the union of two well-colored balanced complete bipartite graphs, of respective sizes $L_{1}$ and $L_{3}$. We distinguish two cases:

* $L_{1} \not \equiv L_{3} \bmod [3]$.

Now suppose that either $L_{1} \bmod [3]$ or $L_{3} \bmod [3]$ is equal to 1 . Without loss of generality, assume that $L_{1} \equiv 1 \bmod [3]$. Then from Lemma 2.1 and Remark 1, we reduce the left part to a single clashing stone. If $L_{3}=0$, then it is done. Otherwise, we know that $L_{3}$ is at least equal to 2. By playing as shown in Figure 4, we reduce the graph to a well-colored balanced complete bipartite of length $L_{3}-1$. Since $L_{3}-1 \not \equiv 0 \bmod [3]$ and from Lemma 2.1, this resulting graph is 1-reducible.

Now suppose that neither $L_{1} \bmod [3] \operatorname{nor} L_{3} \bmod [3]$ is equal to 1 . Therefore and without loss of generality, we can assume $L_{1} \equiv 2 \bmod [3]$ and $L_{3} \equiv 0 \bmod [3]$. By Lemma 2.1, we reduce the left part to a single non clashing stone. If $L_{3}=0$, then it is done. Otherwise, $L_{3} \geq 3$. We proceed as depicted by Figure 4 to reduce the configuration. This operation 


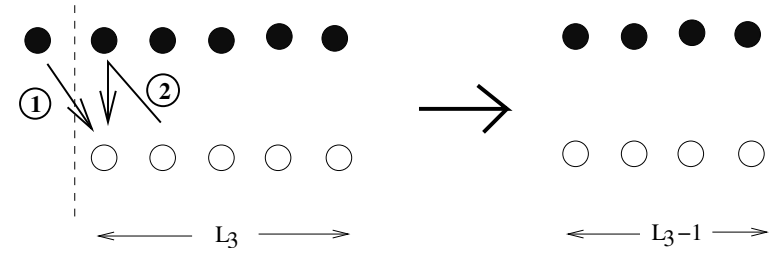

Figure 4. Reduction in a special case.

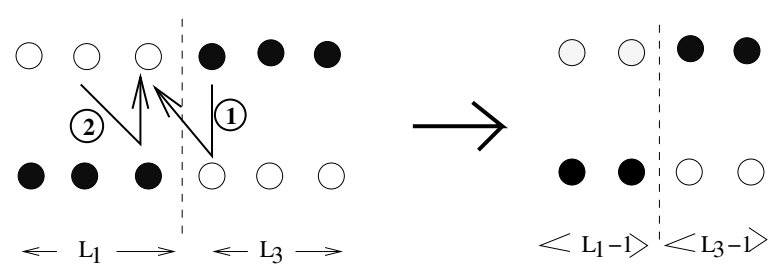

Figure 5 . The case $L_{1} \equiv L_{3} \equiv 0 \bmod [3]$.

yields a well-colored balanced complete bipartite graph of length $\left(L_{3}-1\right)$. Since $\left(L_{3}-1\right) \equiv 2 \bmod [3]$, we conclude to the 1-reducibility thanks to Lemma 2.1.

* $L_{1} \equiv L_{3} \bmod [3]$.

If $L_{1}$ and $L_{3}$ are not multiples of 3, then from Lemma 2.1, both parts are 1-reducible. Therefore, the whole configuration is 2-reducible. If $L_{1} \equiv$ $L_{3} \equiv 0 \bmod [3]$, we play as depicted by Figure 5 .

The resulting configuration is a balanced complete bipartite graph with $L_{1} \equiv L_{3} \equiv 2 \bmod [3]$, which is 2-reducible.

\subsubsection{The reducibility value of non-balanced complete bipartite graphs}

We now consider the general case on $K_{n, m}$ with $\left|S_{0}\right|=n>\left|S_{1}\right|=m>0$. Unlike balanced complete bipartite graphs, we will show that the reducibility value might not be bounded by a constant. For example, it suffices to choose $\left|S_{1}\right|=1$ and $\left|S_{0}\right|>\left|S_{1}\right|$, put a black stone on $S_{1}$ and only white stones on $S_{0}$. In such a configuration, only one move is available, leading to a reducibility value equal to $\left|S_{0}\right|$.

In order to describe a game configuration on $K_{n, m}$, we consider the following set of parameters: The values $n_{b}$ and $n_{w}$ denote respectively the numbers of black and white stones in $S_{0}$. Obviously we have $n=n_{b}+n_{w}$. Similarily, $m_{b}$ and $m_{w}$ denote respectively the numbers of black and white stones in $S_{1}$.

Without loss of generality, we consider configurations satisfying $n_{b} \leq n_{w}$. Under this condition, we define a nonnegative integer $q=n_{w}-n_{b}$.

With these parameters, a game configuration belongs to one of these two forms:

(F1): Configurations for which $q-m<m_{b}$.

(F2): Configurations for which $q-m \geq m_{b}$. 


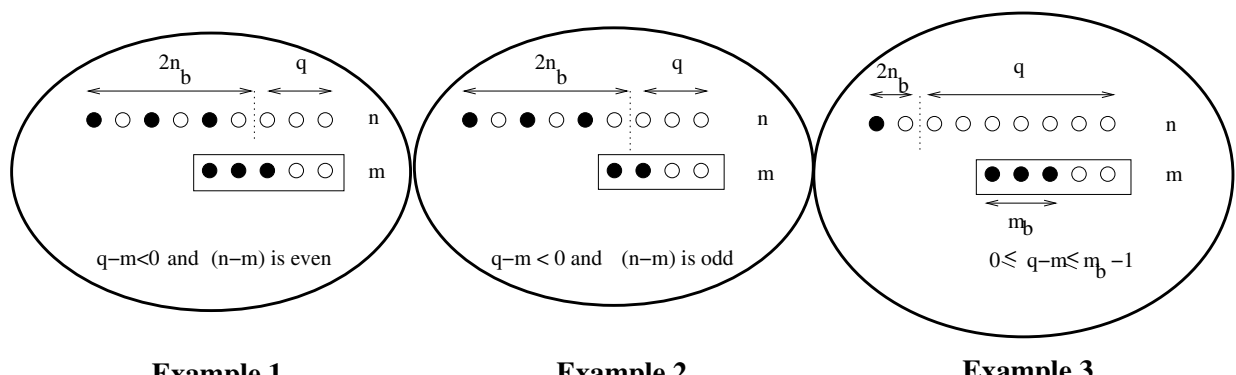

$\begin{array}{lll}\text { Example 1 } & \text { Example 2 } & \text { Example 3 }\end{array}$

Figure 6 . Three examples of configurations in the form $(F 1)$.

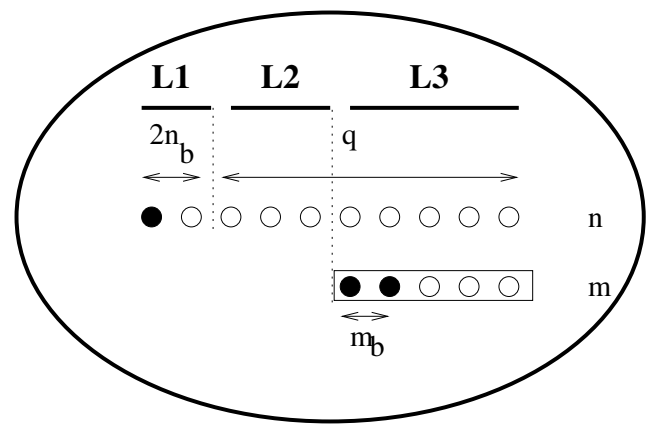

FiguRE 7. A configuration in the form $(F 2)$.

Figure 6 illustrates the form $(F 1)$ through three examples, according to the sign of $(q-m)$ and the parity of $(n-m)$. The subdivision into these cases will become clear later.

Figure 7 illustrates the second form. In that case, the stable set $S_{0}$ can be partitioned into three parts:

$L_{1}$ contains as many black stones than white ones and satisfies $\left|L_{1}\right|=2 n_{b}$.

$L_{2}$ and $L_{3}$ are monochromatic parts, of respective sizes $(q-m)$ and $m$.

We will prove that configurations in the form $(F 1)$ have a reducibility value equal to 1 or 2 . The results will be similar to the "balanced case". For the configurations in the form $(F 2)$, we will present a formula depending on the values $q, m$ and $m_{b}$. For such configurations, we introduce new results that will help us to conclude.

Let $C_{2}$ be a game configuration in the form $(F 2)$. We define the integer function $f$ as:

$$
f\left(C_{2}\right)=q-m-m_{b} .
$$

By definition of $(F 2)$, we have $f\left(C_{2}\right) \geq 0$. 
The following lemma asserts that $f$ never decreases during the game. Moreover, it adds that any move from a configuration in $(F 2)$ yields a resulting configuration also in $(F 2)$.

Lemma 2.3. Let $C_{2}$ be a game configuration in (F2), and let $R$ be the resulting configuration after an arbitrary move from $C_{2}$. If $R$ is not a stable set, then $R$ is also in (F2) and satisfies $f\left(C_{2}\right) \leq f(R)$.

Proof. Denote by $q^{\prime}, m^{\prime}$ and $m_{b}^{\prime}$ the values of $q, m$ and $m_{b}$ after a move from $C_{2}$ to $R$. These values are defined since $R$ is supposed to be a configuration on a complete bipartite graph.

There are four moves to consider:

1. One play a black stone from $S_{0}$ to $S_{1}$. Then $m_{b}^{\prime}=m_{b}+1, q^{\prime}=q+1$ and $m$ is unchanged. This implies $f(R)=f\left(C_{2}\right)$.

2. One play a white stone from $S_{0}$ to $S_{1}$. We get $q^{\prime}=q-1, m_{b}^{\prime}=m_{b}-1$, and $m$ is unchanged. We still have $f(R)=f\left(C_{2}\right)$.

3. One play a black stone from $S_{1}$ to $S_{0}$. Then $m_{b}^{\prime}=m_{b}-1, m^{\prime}=m-1$, and $q^{\prime}=q-2$. Therefore $f(R)=f\left(C_{2}\right)$.

4. One play a white stone from $S_{1}$ to $S_{0}$. Then $m^{\prime}=m-1, q^{\prime}=q+2$ and $m_{b}$ is unchanged. Therefore $f(R)=f\left(C_{2}\right)+3$.

Moreover, since $f$ is monotonic, we clearly have $q^{\prime}-m^{\prime} \geq m_{b}^{\prime}$, which means that $R$ is in the form $(F 2)$.

We now have collected all the necessary results to prove our main theorem about the reducibility of $K_{n, m}$.

Theorem 2.1. Let $C$ be a game configuration on $K_{n, m}$ with $n>m>0$.

If $q-m<m_{b}$, i.e., $C$ is in $(F 1)$, then $C$ is 1 -reducible if $\delta(C) \not \equiv 0 \bmod [3]$, and 2-reducible otherwise.

If $q-m \geq m_{b}$, i.e., $C$ is in (F2), then $C$ has a reducibility value equal to $\left(q-m-m_{b}+2\right)$.

Proof. C is in the form (F1). We will prove that we can reduce $C$ to a nonmonochromatic balanced complete bipartite graph, so as to apply Lemma 2.2. We distinguish three cases (see Fig. 6).

* $q-m<0$ and $(n-m)$ is even. Let $s$ be any stone of $S_{1}$. Since $(n-m)$ is even, this means that we can play exactly $(n-m)$ stones (precisely $(n-$ $m) / 2$ black stones, and $(n-m) / 2$ white ones) from $S_{0}$ to $s$ by alternating black and white moves. The resulting configuration $R$ is thus a balanced complete bipartite graph. Note that $S_{1}$ is unchanged after this set of operations. Since $q-m<0$, there exists at least a black and a white stone in the stable set $S_{0}$ of $R$, which is consequently not monochromatic.

* $q-m<0$ and $(n-m)$ is odd. As previously, we play exactly $(n-m-1)$ stones (precisely $(n-m-1) / 2$ black stones, and $(n-m-1) / 2$ white ones) from $S_{0}$ to any vertex $s$ of $S_{1}$ by alternating black and white moves. In the resulting configuration $R, S_{0}$ contains exactly $(m+1)$ stones and is not monochromatic, whereas $S_{1}$ is unchanged. We now play any move from 
$S_{0}$ to $S_{1}$. This is possible since $S_{0}$ is not monochromatic. This operation yields a non-monochromatic balanced $K_{m, m}$.

$* q-m \geq 0$. We first play $2 n_{b}$ stones (precisely $n_{b}$ stones of each color) from $S_{0}$ to any vertex $s$ of $S_{1}$ by alternating white and black moves. We then play $(q-m)$ white stones from $S_{0}$ to $S_{1}$. This is possible since $m_{b} \geq(q-m+1)$ for a configuration in $(F 1)$. The resulting configuration $R$ contains at least a black stone in $S_{1}$, and at least a white one in $S_{0}$. Moreover, $R$ is balanced.

In each of these three cases, we yield a proper configuration $R$ which is balanced. We then apply Lemma 2.2 on $R$ to conclude.

C is in the form (F2). We first prove that there exists a way of play that leaves exactly $\left(q-m-m_{b}+2\right)$ stones on the graph.

If $m_{b}>0$, we play the $2 n_{b}$ stones from $L_{1}$ to any vertex $s$ of $S_{1}$ by alternating white and black moves. After this operation, $S_{0}$ is monochromatic (of color white). We then play $\left(m_{b}-1\right)$ white stones from $L_{2}$ to $S_{1}$. The single remaining black stone (which belongs to $S_{1}$ ) then walks in zigzags to clobber all the white stones of $L_{1}$ and $S_{1}$. The resulting configuration is a stable set containing a black stone and $\left(q-m-m_{b}+1\right)$ white ones (coming from $\left.L_{2}\right)$.

If $m_{b}=0$, then $L_{1}$ is not empty, since we do not consider monochromatic configurations. Therefore, we play a black stone from $L_{1}$ to $S_{1}$. We get a resulting configuration $C^{\prime}$ in $(F 2)$ with parameters $m_{b}^{\prime}=m_{b}+1$ and $q^{\prime}=q+1$. We now apply the same method as above, which leaves $\left(q^{\prime}-m-m_{b}^{\prime}+2\right)=\left(q-m-m_{b}+2\right)$ stones.

We now prove that any succession of moves leaves at least $\left(q-m-m_{b}+2\right)$ stones on the graph.

On a configuration $C$ in $(F 2)$, we consider a succession of moves that leaves a minimum number of stones. Denote by $C^{\prime}$ the final configuration after such a way of play. No move is playable from $C^{\prime}$.

We first suppose that $C^{\prime}$ is not a stable set. According to Lemma 2.3, $C^{\prime}$ belongs to $(F 2)$ and satisfies $f\left(C^{\prime}\right) \geq f(C)$. Denote by $q^{\prime}, m^{\prime}, m_{b}^{\prime}, L_{1}^{\prime}, L_{2}^{\prime}, L_{3}^{\prime}$ the parameters previously defined and relative to $C^{\prime}$.

Since $m^{\prime}>0, L_{3}^{\prime}$ is not empty, which means that there exists a white stone on $S_{0}$. Since no move is playable from $C^{\prime}$, the set $S_{1}$ is monochromatic of color white, i.e., $m_{b}^{\prime}=0$, and $L_{1}^{\prime}$ is empty.

Moreover, the monotonicity of the function $f$ ensures that

$$
q^{\prime}-m^{\prime}-m_{b}^{\prime}=q^{\prime}-m^{\prime} \geq q-m-m_{b} .
$$

Since there is at least a stone in $L_{3}^{\prime}$ and another one in $S_{1}$, the number of remaining stones on $C^{\prime}$ is at least $\left|L_{2}^{\prime}\right|+2$. Then $\left|L_{2}^{\prime}\right|+2=q^{\prime}-m^{\prime}+2 \geq q-m-m_{b}+2$ according to (1).

Now assume that $C^{\prime}$ is a stable set. This means that the last move of the optimal way of play was done from $S_{1}$ to $S_{0}$. It remains optimal if we consider this last move in the opposite direction (i.e., from $S_{0}$ to $S_{1}$ ). Now consider this 


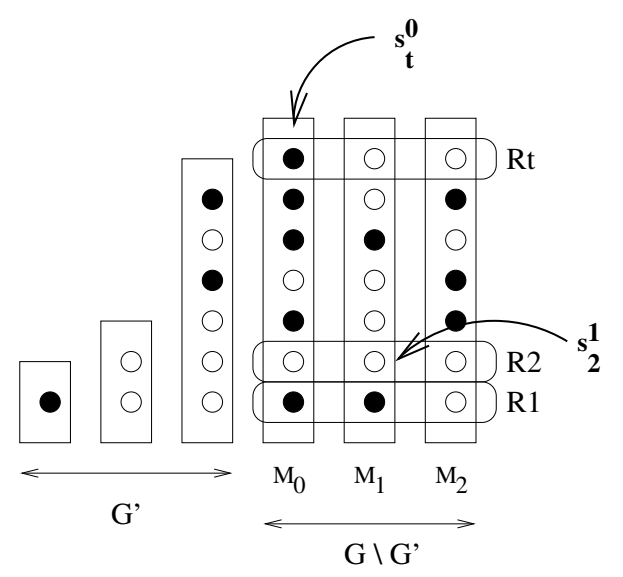

Figure 8. An example of a configuration on a $P$-partite graph $(P=6$ and $i=3)$.

new optimal way of play where $C^{\prime}$ is not a stable set and refer to the previous case to conclude.

\subsection{ISC PlAyed ON COMPlete MUltipartite GRAPHS (With AT LEAST three PARTS)}

Let $P>2$. Denote by $S_{0}, S_{1}, \ldots, S_{P-1}$ the $P$ stable sets of a complete $P$-partite graph $G$. Among these $P$ stable sets, denote by $M_{0}, \ldots, M_{i-1}$ the $i$ stable sets whose size is maximum, i.e., $\left|M_{j}\right| \geq\left|S_{l}\right|$ for all $j=0 \ldots i-1$ and $l=0 \ldots P-1$. We call them the maximum stable sets of $G$.

Denote by $G^{\prime}$ the induced subgraph $G \backslash\left\{M_{0}, \ldots, M_{i-1}\right\}$. For more convenience, we graphically define "rows" of stones on $G \backslash G^{\prime}$ : if $t=\left|M_{0}\right|=\ldots=\left|M_{i-1}\right|$, then a row $R_{j}$ with $1 \leq j \leq t$ defines a set of $i$ stones (one stone per maximum stable set) such that $\bigcup_{1 \leq j \leq t} R_{j}$ is the set of all the stones belonging to $G \backslash G^{\prime}$. Of course, the number of partitions of this form is very large (each new "graphical" arrangement of the stones in $G \backslash G^{\prime}$ defines a new set of rows).

Finally, denote by $s_{j}^{k}$ (with $1 \leq j \leq t$ and $0 \leq k<i$ ) the vertex that both belongs to $M_{k}$ and $R_{j}$.

Figure 8 illustrates these notations.

The reducibility value of $P$-partite graphs depends on the number $i$ of maximum stable sets. When $i>1$, any game configuration is 1-reducible. The reducibility value may be far larger when there is a unique maximum stable set.

Theorem 2.2. Let $C$ be a game configuration on a $P$-partite graph (with $P>2$ ) having at least two maximum stable sets. Then $C$ is 1-reducible.

Proof. Denote by $t$ the value $\left|M_{0}\right|$, i.e. the size of each maximum stable set.

If $t=1$, then the graph is a clique, which is clearly 1-reducible (see [6] for more details). 


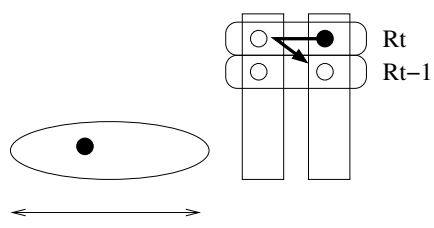

G'

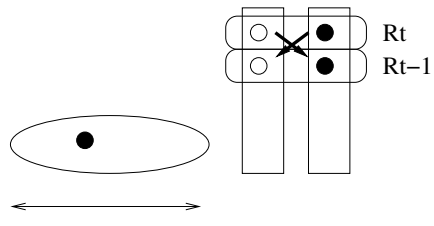

$\mathrm{G}^{\prime}$

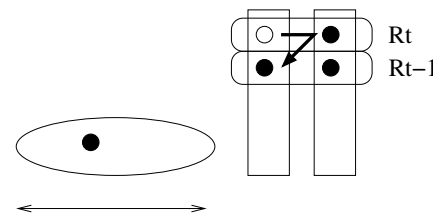

G'

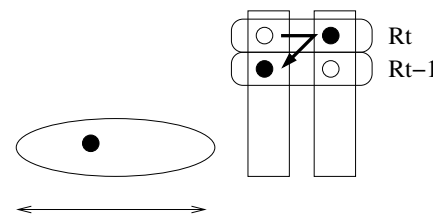

$\mathrm{G}^{\prime}$

Figure 9. Playing when $i=2$ and $G \backslash G^{\prime}$ is not monochromatic.

Now consider $t>1$ and assume that the result is true for all $1 \leq t^{\prime}<t$. We consider three cases:

1. $G \backslash G^{\prime}$ is monochromatic of color $c$. Since $G$ is not monochromatic, there exists a vertex $x$ in $G^{\prime}$ whose color is $\bar{c}$. Now have $x$ clobber the monochromatic row $R_{t}$, and then any stone of the row $R_{t-1}$. In the resulting nonmonochromatic configuration, the size of the maximum stable sets is $(t-1)$, so that we can apply the induction hypothesis.

2. $G \backslash G^{\prime}$ is not monochromatic and $i \geq 3$. We consider a partition of $G \backslash G^{\prime}$ into $t$ rows, such that $R_{t}$ is not monochromatic. Such a partition is possible since $G \backslash G^{\prime}$ is not monochromatic. Besides, since $i \geq 3$, there exists a stone of $R_{t}$ whose color is not rare in $R_{t}$. Without loss of generality, assume that $s_{t}^{0}$ satisfies this property. For the same reasons, either $s_{t-1}^{1}$ or $s_{t-1}^{2}$ has a common color. Assume that $c\left(s_{t-1}^{1}\right)$ is not rare.

The row $R_{t}$ is now seen as a clique. From [6], we know that cliques are strongly 1-reducible, which means that we can choose the location and the color of the unique remaining stone (provided some conditions are fulfilled, which will be the case here). Hence we reduce $R_{t}$ to a single stone of color $\overline{c\left(s_{t-1}^{1}\right)}$ and located on $s_{t}^{0}$. We then play from $s_{t}^{0}$ to $s_{t-1}^{1}$, the resulting configuration is not monochromatic, so that we can apply the induction hypothesis.

3. $G \backslash G^{\prime}$ is not monochromatic and $i=2$. As previously, choose a partition into $t$ rows such that $R_{t}$ is not monochromatic. Since $P>2$, at least a color, say black, appears in $G^{\prime}$. According to the colors of $R_{t-1}$, we play as follows to get a non monochromatic resulting graph, whose size of the maximum stable sets is $(t-1)$. We show 1-reducibility by applying the induction hypothesis. 


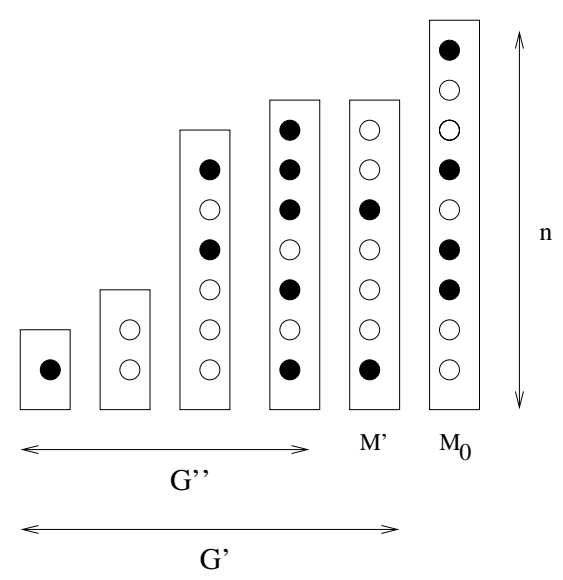

Figure 10. An example of a $P$-partite graph with an unique maximum stable set.

When the graph admits an unique maximum stable set $M_{0}$ of size $t$, we define the values $n=t, m$ as the number of stones in $G^{\prime}$ and $m_{b}$ the number of black stones in $G^{\prime}$. The values $n_{b}$ and $n_{w}$ are respectively the numbers of black and white stones in $M_{0}$. Without loss of generality, assume that $n_{b} \leq n_{w}$. We thus define $q$ as the value $n_{w}-n_{b}$. We denote by $M^{\prime}$ a stable set of $G^{\prime}$ whose size is maximum (i.e, $M^{\prime}$ is a "second maximum" stable set in $G$ ). Denote by $m^{\prime}$ the size of $M^{\prime}$. Finally, denote by $G^{\prime \prime}$ the graph $G^{\prime} \backslash M^{\prime}$. Figure 10 shows a $P$-partite graph with an unique maximum stable set.

Using these notations, we extend the function $f$ to a complete $P$-partite graph having an unique maximum stable set (with $P \geq 2$ ). We define $f$ as $f(C)=$ $q-m-m_{b}$. Lemma 2.3 is thus extended to $P$-partite configurations:

Lemma 2.4. Let $C$ be a game configuration on a complete $P$-partite graph having an unique maximum stable set (with $P>2$ ) satisfying $q-m \geq m_{b}$, and let $R$ be the resulting configuration after an arbitrary move from $C$. Then $R$ is also $P$-partite (with $P \geq 2$ ), has an unique maximum stable set and the inequality $f(C) \leq f(R)$ holds.

Proof. If is straightforward to see that $R$ is $P$-partite with $P \geq 2$.

By hypothesis, the configuration $C$ satisfies

$$
q=n-2 n_{b} \geq m+m_{b}=m_{b}+\left|M^{\prime}\right|+\left|G^{\prime \prime}\right| \geq m_{b}+\left|M^{\prime}\right|+1 .
$$

Hence we have

$$
n \geq m_{b}+2 n_{b}+\left|M^{\prime}\right|+1 .
$$

Since $C$ is a proper configuration, the values $m_{b}$ and $n_{b}$ can not be simultaneously equal to 0 . This implies that $n \geq\left|M^{\prime}\right|+2$. Therefore, any move from $C$ leads to a configuration $R$ where $M_{0}$ remains the unique maximum stable set. This result ensures that the function $f$ is defined on $R$. 
If we consider any move between $G^{\prime}$ and $M_{0}$, then we refer to Lemma 2.3 to assert that $f(C) \leq f(R)$. And if we consider a move inside of $G^{\prime}$, we remark that $q$ is unchanged and the values $m$ and $m_{b}$ are not increased. This also implies $f(C) \leq f(R)$.

Theorem 2.3. Let $G$ be a $P$-partite graph (with $P>2$ ) having an unique maximum stable set. Any game configuration on $G$ such that $q-m<m_{b}$ is 1-reducible. Any game configuration on $G$ such that $q-m \geq m_{b}$ has a reducibility value equal to $q-m-m_{b}+2$.

\section{Proof. Any game configuration satisfying $q<m$ is 1-reducible.}

According to $n$, we choose a "minimal" counter-example $C$, i.e. $C$ satisfies $q<m$, is not 1-reducible, and there is no other configuration of this type whose size of $M_{0}$ is smaller than $n$.

We first suppose that $n_{b} \neq 0$ and we consider three cases for $C$ :

$* n-m^{\prime}=1$. We play any authorized move from $M_{0}$ to $M^{\prime}$ : this is possible since $n_{w} \geq n_{b}>0$. Therefore, the resulting configuration is not monochromatic, and admits at least two maximum stable sets of size $n-1\left(M_{0}\right.$ and $M^{\prime}$ ). From Theorem 2.2, it is 1-reducible, which ensures the contradiction.

$* n-m^{\prime} \geq 2$ and $n_{b} \geq 2$. We play successively a black and a white stone from $M_{0}$ to any stone of $G^{\prime}$. The resulting configuration is not monochromatic since there remains at least a stone of each color in $M_{0}$, and still satisfies $q<m$ (both values are unchanged). If $\left|M_{0}\right|=\left|M^{\prime}\right|$ after the operation, then it is 1-reducible from Theorem 2.2. If not, this means that $M_{0}$ remains the unique maximum stable set, but its size is now smaller than $n$, which yields a contradiction by minimality of $C$.

* $n-m^{\prime} \geq 2$ and $n_{b}=1$. If $m_{b}>0$, we play any white stone from $M_{0}$ to any black one of $G^{\prime}$. The resulting configuration is not monochromatic, has an unique maximum stable set, still satisfies $q<m$ (since $m$ is unchanged and $q$ is decreased by 1 ), and is smaller than $C$. It thus contradicts the minimality of $C$. Hence we have $m_{b}=0$, which means that $G^{\prime}$ is monochromatic white. With the unique black stone of the configuration, we clobber alternately stones of $M_{0}$ and $G^{\prime \prime}$, until $M^{\prime}$ and $M_{0}$ have the same size. This is possible, since the number of stones in $G^{\prime \prime}$ is equal or greater than $q+1-m^{\prime}$. Note that in the case where $\left|G^{\prime \prime}\right|$ exactly equals $q+1-m^{\prime}$, the black stone will delete the entire $G^{\prime \prime}$ and then alternately clobber all the white stones between $M^{\prime}$ and $M_{0}$. In any case, we reduce $C$ to a single stone, which contradicts its existence.

Therefore $C$ satisifies $n_{b}=0$. We consider two cases:

* $G^{\prime}$ is monochromatic black. Then play any white stone from $M_{0}$ to $G^{\prime}$. The resulting configuration is not monochromatic (since there remains at least a black stone in $G^{\prime}$ ), and still satisfies $q<m$ ( $m$ is unchanged and $q$ is decreased by 1). In the resulting configuration, if $M_{0}$ and $M^{\prime}$ have the same size, we conclude to 1-reducibility thanks to Theorem 2.2. Otherwise, 
$M_{0}$ is the unique maximum stable set and it contradicts the minimality of $C$.

* $G^{\prime}$ is not monochromatic. Let $s$ be any black stone of $G^{\prime}$. Then $s$ clobers any white stone of $M_{0}$ and then any other white stone of $G^{\prime}$. The parameters $q$ and $m$ are both decreased by one after these moves. Once again, the minimality of $C$ is contradicted. Note that the resulting configuration contains is still $P$-partite with $P \geq 3$. Indeeed, if it was bipartite, this would mean that $C$ contains at least two maximum stable sets, since $q<m$.

Any game configuration satisfying $0 \leq q-m<m_{b}$ is 1-reducible.

As previously, we choose $C$ as a minimal configuration satisfying $0 \leq q-m<$ $m_{b}$, not 1-reducible, and such that there is no other configuration of this type whose size of $M_{0}$ is smaller than $n$. Note that this implies $m_{b}>0$.

We first suppose that $n_{b} \neq 0$. As previously, if $n-m^{\prime}=1$, we play an authorized move from $M_{0}$ to $G^{\prime}$, so as to apply Theorem 2.2. If $n-m^{\prime}>1$, we play successively a black and a white stone from $M_{0}$ to any stone of $G^{\prime}$ : the resulting configuration $R$ has its parameters $q, m$ and $m_{b}$ unchanged, and is not monochromatic, since $m_{b} \geq 1$ and there are at least $m>0$ white stones in $M_{0}$. As previously, we either conlude to the 1-reducibility with Theorem 2.2 (if $\left|M_{0}\right|=\left|M^{\prime}\right|$ in $R$ ), or to the non-minimality of $C$.

Hence $C$ satisfies $n_{b}=0$. This means that $\left|M_{0}\right|$ is monochromatic white. We consider two cases:

* $G^{\prime}$ is not monochromatic black.

If $G^{\prime}$ contains at least three non empty stable sets, then play any black stone $s$ from $G^{\prime}$ to any white stone of $M_{0}$ and then to any white stone of $G^{\prime}$. The resulting configuration $R$ is still $P$-partite with $P \geq 3$, and is not monochromatic (there is at least a black stone in $G^{\prime}$ and a white one in $M_{0}$ ). Besides, $q$ and $m$ are both decreased by 1 , and $m_{b}$ is unchanged, which implies that we still have $0 \leq q-m<m_{b}$. We either conlude to the 1-reducibility thanks to Theorem 2.2 (if $\left|M_{0}\right|=\left|M^{\prime}\right|$ in $R$ ), or to the non-minimality of $C$.

If $G^{\prime}$ contains exactly two stable sets and if $m_{b} \geq 2$, we apply the same method by choosing a black stone $s$ such that $s$ does not belong to a stable set of size one. It is always possible to find such a stone, since there are at least three stones in $G^{\prime}$ (two blacks and a white) and only two stable sets. With this choice, the resulting configuration remains tripartite.

If $G^{\prime}$ contains exactly two stable sets and if $m_{b}=1$, this implies $q=m$. There are as many stones in $G^{\prime}$ than in $M_{0}$. We use the only black stone to clobber alternately the white stones of $M_{0}$ and $G^{\prime}$. This yields a unique stone on the graph in the end.

* $G^{\prime}$ is monochromatic black.

If $q>m$, then play any white stone from $M_{0}$ to $G^{\prime}$. After this operation, $q$ and $m_{b}$ are decreased by 1 and $m$ is unchanged. Hence we still have $0 \leq q-m<m_{b}$. The resulting configuration $R$ is not monochromatic, 
since there are at least a black and a white stone in $G^{\prime}$. We either conlude to the 1-reducibility with Theorem 2.2 (if $\left|M_{0}\right|=\left|M^{\prime}\right|$ in $R$ ), or to the non-minimality of $C$.

If $q=m$, we ignore the edges inside $G^{\prime}$ and see $C=G^{\prime} \cup M_{0}$ as a wellcolored balanced complete bipartite graph. From Lemma 2.2, if $\delta(C) \not \equiv$ $0 \bmod [3]$, then $C$ is 1-reducible. And if $\delta(C) \equiv 0 \bmod [3]$, any white stone of $M_{0}$ clobbers successively two black stones of $G^{\prime}$ : this operation uses an inside edge of $G^{\prime}$, and thus changes the value of $\delta(C) \bmod [3]$. Thanks to Lemma 2.2, we conclude to the 1-reducibility of $C$.

Any game configuration satisfying $q-m \geq m_{b}$ has a reducibility value equal to $q-m-m_{b}+2$.

Let $C$ be a game configuration satisfying $q-m \geq m_{b}$. If we ignore the edges inside of $G^{\prime}, C$ can be seen as a bipartite graph $G^{\prime} \cup M_{0}$, where $M_{0}$ is the largest stable set. The parameters $q, m$ and $m_{b}$ have been extended from bipartite to multipartite graphs. From Theorem 2.1 about complete bipartite graphs, we conlude that the reducibility value of $C$ is at most $q-m-m_{b}+2$.

We now consider $C^{\prime}$, a final configuration after having played an optimal succession of moves from $C$. Note that $C^{\prime}$ is necessarily monochromatic. If $C^{\prime}$ is $P$-partite with $P \geq 2$, denote by $m^{\prime}, n^{\prime}, n_{b}^{\prime}, m_{b}^{\prime}$ and $q^{\prime}$ the parameters of $C^{\prime}$. We study two cases.

If $C^{\prime}$ is $P$-partite with $P \geq 3$, then without loss of generality, assume that $n_{b}^{\prime}=m_{b}^{\prime}=0$ (since $C^{\prime}$ is monochromatic). The number of remaining stones on $C^{\prime}$ is at least $\left|M_{0}\right|+\left|G^{\prime}\right| \geq n^{\prime}+2=q^{\prime}+2$. Since $f$ is monotonic from Lemma 2.4, we have $q^{\prime}-m^{\prime}-m_{b}^{\prime}=q^{\prime}-m^{\prime}=f\left(C^{\prime}\right) \geq f(C)=q-m-m_{b}$. Hence the number of remaining stones is at least $q^{\prime}+2 \geq q-m-m_{b}+m^{\prime}+2 \geq q-m-m_{b}+2$.

If $C^{\prime}$ is $P$-partite with $P<3$, then there exists a configuration $C^{\prime \prime}$ on a complete bipartite graph in the optimal way of play from $C$ to $C^{\prime}$. Since $C^{\prime \prime}$ satisifes $q-m \geq m_{b}$ (according to Lem. 2.4), we can apply Theorem 2.1 to prove that the reducibility value of $C^{\prime \prime}$ is greater than $q-m-m_{b}+2$.

\section{Extremal QUeStions}

Let us denote by $\operatorname{rv}(G, C)$ the reducibility value of $G$ with configuration $C$, and denote by $\operatorname{maxrv}(G)$ the maximum of these values taken over all proper configurations, and $\operatorname{minrv}(G)$ the minimum of these values taken over the same set.

The purpose of this section is to estimate the values of $\operatorname{minrv}(G)$ and $\operatorname{maxrv}(G)$ for all graph $G$.

\subsection{VAlue of $\operatorname{minrv}(G)$}

We determine the exact value of $\operatorname{minrv}(G)$ for all graph $G$, and prove that it is simply equal to the number of connected components of $G$.

Lemma 3.1. Let $T$ be a tree. Then we have

$$
\operatorname{minrv}(T)=1 .
$$


Proof. The proof works by induction on the number of vertices of $T$. If $T$ has one vertex, then the statement is trivial. If $T$ has at least two vertices, then let $u$ be a leaf of $T$. By induction, the tree $T^{\prime}:=T \backslash\{u\}$ admits a configuration $C^{\prime}$ such that $\operatorname{rv}\left(T^{\prime}, C^{\prime}\right)=1$. Let $v$ be the unique neighbour of $u$ in $T$, and let $C$ be the following configuration on $T$ :

* $C(w)=C^{\prime}(w)$ for all $w \neq u, v$;

* $C(u)=C^{\prime}(v)$;

* $C(v)=$ black if $C^{\prime}(v)=$ white, and $C(v)=$ white otherwise.

It is easy to check that $\operatorname{rv}(T, C)=1$ (hint: the first move is $u$ clobbering $v$ ).

Theorem 3.1. Let $G$ be a connected graph. Then we have

$$
\operatorname{minrv}(G)=1
$$

Proof. This is a straightforward consequence of Lemma 3.1: since $G$ is connected, then it admits a spanning tree $T$, that is a tree which is a subgraph of $G$ having the same vertex set as $G$. Lemma 3.1 implies the existence of a configuration $C$ such that $\operatorname{rv}(T, C)=1$, which implies $\operatorname{rv}(G, C)=1$.

\subsection{Bounds On $\operatorname{maxrv}(G)$}

\subsubsection{Upper bound}

We prove an upper bound related to the minimum degree of the graph, and we characterize the graphs for which this bound is tight.

Theorem 3.2. Let $G$ be a graph on $n$ vertices of minimum degree $\delta$, and let $C$ be a proper configuration of $G$. Then we have

$$
\operatorname{rv}(G, C) \leq n-\delta
$$

Furthermore, one can make at least $\delta$ moves by, step by step, greedily choosing any move which leaves at least one connected component with a proper configuration.

Proof. The proof works by induction on $\delta$. If $\delta=1$, then there exists at least an edge $u v$ such that there are stones of different colors on $u$ and $v$, hence one can make at least one move. If $\delta \geq 2$, then there exists at least an edge $u v$ such that there are stones of different colors on $u$ and $v$. Playing along this edge (either $u$ clobbering $v$ or $v$ clobbering $u$ ) surely reduces to a graph of minimum degree at least $\delta-1$, but the graph may not be connected, and the configurations on the connected components of this graph may not be proper. However, it is enough to get at least one connected component whose configuration is proper to apply the induction hypothetis. Now two cases follow.

If playing along the edge $u v$ (either $u$ clobbering $v$ or $v$ clobbering $u$ ) leads to a new graph for which at least one connected component $C$ has a proper configuration, then we can apply the induction hypothesis on $C$. If not, then let us assume that the stone on $u$ is black (and then the stone on $v$ is white). The fact that 


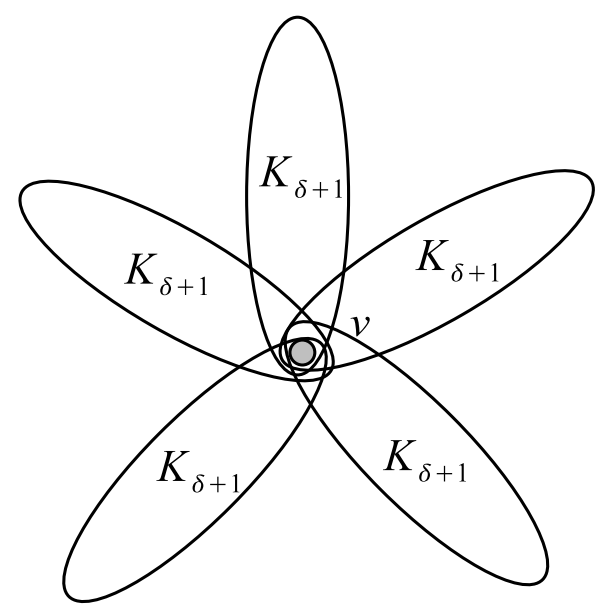

FIGURE 11. The graph $S_{k}\left(K_{\delta+1}\right)$.

either $u$ clobbering $v$ or $v$ clobbering $u$ does not lead to a graph for which at least one its connected component has a proper configuration implies the following:

* the edge $u v$ is such that $G \backslash\{u v\}$ has two connected components $U$ and $V$, such that $u \in U$ and $v \in V$;

* all the stones on vertices of $U \backslash\{u\}$ are white;

* all the stones on vertices of $V \backslash\{v\}$ are black.

In this case, one can still apply the induction hypothesis by, for instance, clobbering a stone of $U$ with the stone placed on $u$.

For any $\delta \geq 1$, let us define $\mathcal{G}_{\delta}$, a set of connected graphs of minimum degree $\delta$ equipped with proper configurations, as follows:

* the complete graph on $\delta+1$ vertices equipped with any proper configuration belongs to $\mathcal{G}_{\delta}$ for all $\delta \geq 1$

* for any integer $k \geq 2$, let us define $S_{k}\left(K_{\delta+1}\right)$ as the non-disjoint union of $k$ copies of $K_{\delta+1}$, where one and only one vertex $v$ belongs to all $k$ copies of $K_{\delta+1}$, and no other vertex belongs to more than one copy of $K_{\delta+1}$ (one can think of $S_{k}\left(K_{\delta+1}\right)$ as a star $K_{1, k}$, where each of the $k$ leaves has been expanded into a $K_{\delta}$, see Fig. 11). Then $S_{k}\left(K_{\delta+1}\right)$, equipped with a configuration such that the color of the vertex $v$ is rare, belongs to $\mathcal{G}_{\delta}$ for all $k \geq 2$;

* for $\delta=2$, the cycle on 4 vertices equipped with any configuration containing only two black stones, which are placed on consecutive vertices of the cycle, belongs to $\mathcal{G}_{2}$;

* no other graph belongs to $\mathcal{G}_{\delta}$. 
Theorem 3.3. For all $\delta \geq 1$, the set of connected graphs $G$ having minimum degree $\delta$ equipped with a proper configuration $C$ such that

$$
\operatorname{rv}(G, C)=n-\delta
$$

is exactly $\mathcal{G}_{\delta}$.

Proof. The proof works by induction on $\delta$. The case $\delta=1$ is easy to prove. Take an edge $u v$ such that a black stone lies on $u$ and a white stone lies on $v$. Assume that $u$ has other neighbours than $v$. Since the reducibility value is $n-1$, then all the other neighbours of $u$ are white, and form a stable set. This implies that $v$ has no other neighbours than $u$.

Now let $\delta \geq 2$, and let $G$ be a connected graph on $n$ vertices having minimum degree $\delta$ equipped with a proper configuration $C$ such that $\operatorname{rv}(G, C)=n-\delta$. Let $u v$ be the edge along which the first move of a game ending after exactly $\delta$ moves is made. We may assume that the stone lying on $u$ is black, and that this stone clobbers a white stone placed on $v$. Let $G^{\prime}$ be the graph obtained after this first move. We then claim the following:

The graph $G^{\prime}$ has minimum degree at least $\delta-1$, and all the connected components of $G^{\prime}$ are of cardinality greater than or equal to $\delta$. Moreover, there is one and only one of these components whose configuration is proper.

The first part of the claim is obvious since $G$ has minimum degree $\delta$. The second part derives from Theorem 3.2. Indeed, if $G^{\prime}$ had at least two connected components equipped with proper configurations, then we could make at least $\delta-1$ moves on one, and $\delta-1$ moves on the other, which is a contradiction with $\operatorname{rv}(G, C)=n-\delta$ since $\delta \geq 2$. Hence claim (2) is true, and this implies the following:

One and only one connected component of $G^{\prime}$ is a member of $\mathcal{G}_{\delta-1}$, and all the others are white monochromatic cliques on $\delta$ vertices.

From claim (2), we know that $G^{\prime}$ has a connected component $A$ equipped with a proper configuration. This component has minimum degree at least $\delta-1$, and since $\operatorname{rv}(G, C)=n-\delta$, then one can actually make exactly $\delta-1$ more moves on $A$, hence $A \in \mathcal{G}_{\delta-1}$. We know from (2) that all the other components are monochromatic, and that they have at least $\delta$ vertices. Let $B$ be such a component. By definition, no vertex of $B$ is adjacent to $v$. This implies that $B$ is white monochromatic. Indeed, if $B$ were black monochromatic, then $v$ could clobber $u$, and this would lead to a graph equipped with a proper configuration of minimum degree $\delta$. By Theorem 3.2, one could make at least $\delta$ more moves, a contradiction with $\operatorname{rv}(G, C)=n-\delta$. Now, let $w$ be a neighbour of $u$ in $B$. The second part of Theorem 3.2 implies that $u$ clobbering $w$ can be the first of a sequence of exactly $\delta$ moves, hence $B$ is a member of $\mathcal{G}_{\delta-1}$. For all $\delta \geq 2$, it is easy to check that the only one possibility is that $B$ is a complete graph on $\delta$ vertices, which proves the second part of (3). 
To complete the proof, it remains then to make a case study by applying (3) and using the induction hypothesis. The cases $\delta=2$ and $\delta=3$ have to be studied separately, because of the sporadic event of the cycle on four vertices belonging to $\mathcal{G}_{2}$. This (rather straightforward) case study is left to the reader.

\subsubsection{Lower bound}

Theorem 3.4. Let $C_{0}$ be a cutset of a simple, connected graph $G$, and let $C_{1}, C_{2}, \ldots$, $C_{k}$ be the connected components of $G \backslash C_{0}$. Let us assume that $\left|C_{0}\right|<k$. Let $C$ be the following configuration of stones on $G$ : a black stone lies on all the vertices of $C_{0}$, and a white stone lies on all the vertices of $C_{1} \cup C_{2} \cup \ldots \cup C_{k}$. Then the reducibility value of $G$ under the configuration $C$ is such that

$$
\operatorname{rv}(G, C) \geq n-\left(\sum_{i=0}^{\left|C_{0}\right|}\left|C_{i}\right|-1\right)
$$

Proof. The theorem is straightforward if $C_{0}$ is a single point. Indeed, in this case, we have a cutvertex $v_{0}$, on which lies the only black stone of the configuration. If this stone does not clobber any white stone, then one may make only one move, consisting in clobbering this stone with a white stone, and the game ends after 1 round. If the black stone clobbers some white stones, then it can clobber only white stones of a certain component $C_{i}$. Indeed, since $v_{0}$ is a cutvertex, then the black stone can visit only one of the components of $G \backslash C_{0}$.

If $C_{0}$ is a nontrivial cutset, then the situation is a little bit more complicated, since a black stone can visit more than one components of $G \backslash C_{0}$. The idea is the following. If a black stone visits more than one component, then it means that, at some points, this stone had to go back through $C_{0}$, hence clobbering a white stone. Since $C_{0}$ contains originally no white stones, it means that black stones of $C_{0}$ had to be clobbered without having visited any component of $G \backslash C_{0}$. Thus a black stone visiting $p$ components implies that $p-1$ black stones did not visit any component, hence, on average, one black stone can visit at most one component.

More formally, given a vertex $v \in C_{0}$, let us define $s(v)$, the score of the black stone placed on $v$, as the number of components of $G \backslash C_{0}$ that this black stone will visit during the game.

Clearly, we have

$$
\sum_{v \in C_{0}} s(v) \leq\left|C_{0}\right|
$$

Now, the desired inequality is derived from (4). Indeed, in the most favorable case, all the white stones of the $\left|C_{0}\right|$ largest components and of $\left|C_{0}\right|$, but for one, were clobbered, hence the $\sum_{i=0}^{\left|C_{0}\right|}\left|C_{i}\right|-1$ in the formula.

Remark 3.1. This bound is tight for the path on $n$ vertices, where the worst configuration is where the central vertex has a rare color (see Fig. 12). 


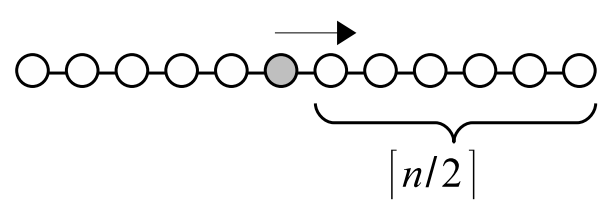

FIGURE 12. The path on $n$ vertices has maximum reducibility value equal to $\left\lceil\frac{n}{2}\right\rceil$.

Indeed, the bound of the previous theorem says that $\operatorname{rv}(G, C) \geq n-\left(1+\left\lfloor\frac{n}{2}\right\rfloor-\right.$ $1)=\left\lceil\frac{n}{2}\right\rceil$, and it is easy to see that $\operatorname{maxrv}\left(\mathcal{P}_{n}\right)=\left\lceil\frac{n}{2}\right\rceil$ (where $\mathcal{P}_{n}$ denotes the path on $n$ vertices). Let us briefly show this. Let $\left\{v_{1}, \ldots, v_{n}\right\}$ denotes the vertex set of $\mathcal{P}_{n}$ such that there is an edge between $v_{i}$ and $v_{i+1}$ for all $i=1, \ldots, n-1$. Let us call 2-block a non-monochromatic interval $v_{i}, \ldots, v_{j}, 1 \leq i<j \leq n$ such that there exists $i \leq k<j$ for which vertices $v_{i}, \ldots, v_{k}$ are of the same color, and vertices $v_{k+1}, \ldots, v_{j}$ are of the same color. Similarly, let us call 3-block a non-monochromatic interval $v_{i}, \ldots, v_{j}, 1 \leq i<j \leq n$ such that there exists $i \leq k<l<j$ for which vertices $v_{i}, \ldots, v_{k}$ are of the same color, vertices $v_{k+1}, \ldots, v_{l}$ are of the same color, and vertices $v_{l+1}, \ldots, v_{j}$ are of the same color. Now partition $\left\{v_{1}, \ldots, v_{n}\right\}$ into a certain number of 2-blocks and one 3-block. Clearly, it is always possible to do this. Now, let us look at a certain 2-block $B$, independently form the other blocks. It is clear that at least $\left\lfloor\frac{|B|}{2}\right\rfloor$ vertices of $B$ can be clobbered by a vertex of $B$. Similarly, at least $\left\lfloor\frac{|B|}{2}\right\rfloor$ vertices of $B$ can be clobbered by a vertex of a given 3-block $B$. Hence, we can always clobber at least $\left\lfloor\frac{n}{2}\right\rfloor$ vertices of $\mathcal{P}_{n}$, which means $\operatorname{maxrv} \mathcal{P}_{n} \leq n-\left\lfloor\frac{n}{2}\right\rfloor=\left\lceil\frac{n}{2}\right\rceil$.

Note that the previous theorem does not apply for the case of cycles, because any cutset has at least as many vertices as the number of remaining components.

\section{REFERENCES}

[1] M.H. Albert, J.P. Grossman, R.J. Nowakowski and D. Wolfe, An introduction to Clobber. INTEGERS $\mathbf{5}$ (2005).

[2] L. Beaudou, E. Duchêne and S. Gravier, A survey about Solitaire Clobber (submitted).

[3] V.D. Blondel, C. de Kerchove, J.M. Hendrickx and R. Jungers, Solitaire Clobber as an optimization problem on words. INTEGERS $\mathbf{7}$ (2008).

[4] B. Bollobás, Random graphs. Cambridge University Press (2001).

[5] E.D. Demaine, M.L. Demaine and R. Fleischer, Solitaire Clobber. Theor. Comput. Sci. 313 (2004) 325-338.

[6] P. Dorbec, E. Duchêne and S. Gravier, Solitaire Clobber played on Hamming graphs. Integers, J. Comb. No. Theory 7 (2008).

[7] A. Itai, C.H. Papadimitriou and J.L. Szwarcfiter, Hamilton paths in grid graphs. SIAM J. Comput. 11 (1982) 676-686.

[8] I. Peterson, Getting clobbered, Science News 161 (2002), http://www.sciencenews.org/ articles/20020427/mathtrek.asp.

[9] M. Ruszinkó, private communication. 